\title{
Evidence Suggesting That Difructose Anhydride III Is an Indigestible and Low Fermentable Sugar during the Early Stages after Ingestion in Humans
}

\author{
Akiko TAMURA $^{1}$, Takuya ShiomI ${ }^{1}$, Norihiro SHIGEMATSU ${ }^{1}$, Fusao TomitA ${ }^{2}$ and Hiroshi HARA ${ }^{2}$ \\ ${ }^{1}$ Central Research Laboratory, FANCL Co., Ltd., 12-13 Kamishinano, Totsuka-ku, Yokohama \\ 244-0806, Japan \\ ${ }^{2}$ Division of Applied Bioscience, Graduate School of Agriculture, Hokkaido University, Sapporo \\ 060-8589, Japan
}

(Received March 4, 2003)

\begin{abstract}
Summary We investigated the influences of difructose anhydride III (DFAIII), a novel commercially available disaccharide, on sugar metabolism, breath hydrogen and serum acetate in the early stages after ingestion to determine whether DFAIII is an indigestible sugar and to what degree it is fermentable in humans. This study was designed as a randomized controlled single-blind crossover test with 9 healthy subjects, who drink a $200 \mathrm{~mL}$ water solution containing $10 \mathrm{~g}$ of DFAIII, lactulose or sucrose following overnight fasting. Blood samples (for analysis of glucose, fructose, insulin, triacylglycerol, free fatty acids, and acetate) were collected at $0,0.5,1,2,4,8 \mathrm{~h}$ after the ingestion and breath samples (for analysis of hydrogen and methane gases) were collected at $1 \mathrm{~h}$ intervals until $8 \mathrm{~h}$ after the ingestion. We also interviewed each subject hourly about the incidence and severity of specific abdominal complaints and other symptoms. The results revealed that ingestion of $10 \mathrm{~g}$ of DFAIII did not change the serum levels of glucose, fructose, and insulin, similarly to the case with lactulose, and no increase in breath hydrogen excretion was comparable to the case with sucrose. The incidence of specific abdominal symptoms tended to be lower after DFAIII ingestion than after lactulose ingestion. It thus turned out that DFAIII was indigestible and low fermentable in the early stages after ingestion.
\end{abstract}

Key Words difructose anhydride III, sugar metabolism, breath hydrogen

Difructose anhydride III (DFAIII; di-D-fructo-furanose $1,2^{\prime}: 2,3^{\prime}$ dianhydride) is a disaccharide which has been isolated from Lycoris radiata (1). The compound has also been found in caramels made from several kinds of sugars $(2,3)$, and commercial roasted chicory (4). Recently, pure DFAIII has been manufactured from inulin by fermentation (5). It has been demonstrated in an in vivo balance study in rats that DFAIII stimulates $\mathrm{Ca}$ absorption from the intestine $(6,7)$ and it also increases net $\mathrm{Ca}$ absorption during in vitro experiments using an Ussing chamber technique $(8,9)$.

Since DFAIII has two glycoside linkages between two fructose moieties (Fig. 1), a unique structure not common to other disaccharides, it is presumed to be less digestible. When indigestible saccharides reach the colon, they are broken down by enterobacteria into not only short-chain fatty acids (SCFA) as represented by acetic, propionic and butyric acid, but also hydrogen, methane or carbon dioxide gases $(10,11)$. Major portions of these fermentation products are absorbed from the colon, which appear somewhat in breath (gases) or

E-mail: aktamura@fancl.co.jp

Abbreviations: DFAIII, di-D-fructo-furanose 1,2': 2,3' dianhydrides; SCFA, short-chain fatty acids; TG, triacylglycerol; FFA, free fatty acids; AUC, area under the curve. peripheral blood (acetate). The fermentability of ingested indigestible saccharides is well reflected in the breath hydrogen level, since enterobacteria alone produce hydrogen excreted in the breath (11). Thus it can be used as an indication of predicting the fermentability of indigestible saccharides in humans (12-21). Although venous acetate appears to be less sensitive than breath hydrogen (17), a stable-isotope method has been used to quantitatively measure acetate production (16), and several studies are based on the blood venous acetate level $(16-18,20,21)$.

In this study, we measured breath hydrogen, methane, and serum acetate to evaluate the fermentability of DFAIII, and blood parameters related to sugar and fat metabolism to evaluate its digestibility. Data was compared to those of lactulose and sucrose.

\section{MATERIALS AND METHODS}

1. Test substances. We used DFAIII (>97\%) manufactured by Nippon Beet Sugar Mfg. Co., Ltd. (Tokyo, Japan) as the test substance. Lactulose of analytical grade ( $>90 \%$, indigestible disaccharide), and sucrose of special grade ( $>99 \%$, digestible disaccharide) were purchased from Wako Pure Chemical Industries (Osaka, Japan) as controls.

2. Subjects. We recruited volunteers from members 


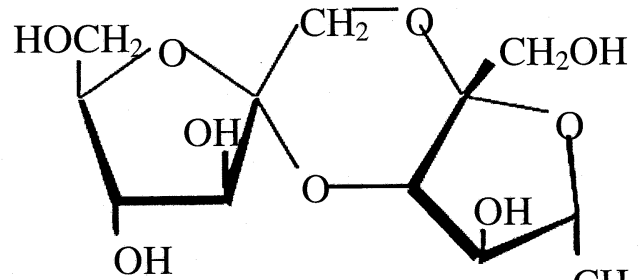

$\mathrm{CH}_{2} \mathrm{OH}$

Fig. 1. Molecular structure of DFAIII subscribed from reference (7).

of the FANCL Co., Ltd, and 10 subjects first agreed to take part in the study. We informed all participants as to the purpose and plan of this study as well as their rights to resign at their own will and our duties to guard their personal privacies against outsiders, from whom the informed consents were obtained in writing. We confirmed by questionnaires that all subjects did neither have any chronic gastrointestinal disorders including diarrhea nor disorders of the viscera (liver, kidney, pancreas, etc.) nor dosage of antibiotics over $10 \mathrm{~d}$ before the start of the study. Prior to the initiation of the study, one male subject was forced to drop out owing to pressure of business. One female subject left the study after the start due to a pregnancy. The remaining 8 subjects were able to follow our experimental protocol without problems. The mean age of the remaining 8 subjects was 28 years old in the range of 23 to 32 .

3. Study design. This study was designed as a randomized controlled single-blind crossover test. Subjects followed the meal plan shown in Table 1, with the last pre-test meal taken at 21:00, on the day before the test. After overnight fasting, blood and breath samples were collected. At 09: 00 the subjects ingested $10 \mathrm{~g}$ of the test or control substances dissolved in $200 \mathrm{ml}$ of water.
Blood samples were collected at $0.5,1,2,4$, and $8 \mathrm{~h}$ after ingestion. Breath samples were collected immediately after gargling with Listerine ${ }^{\circledR}$ (Pfizer Japan Inc., Tokyo, Japan) every hour until $8 \mathrm{~h}$ after ingestion. We interviewed the subjects concerning the presence and severity (none, mild, moderate, severe, very severe) of specific abdominal symptoms (abdominal pain, rumbling in the intestine, distention, nausea, anorexia) and any other symptoms every hour until the post-ingestional $8 \mathrm{~h}$. At the same time, we asked the subjects whether or not any defecation consisted of muddy or watery stools (diarrhea). The subjects were strictly prohibited from eating anything except for a low fiber meal (Table 1) given at 11:00 and 15:00 with free access to drinking water. In addition, they were requested not to undertake excessive exercise, smoke during the test day, or drink alcohol beginning from 3 days before the test day to the finish of the test. Each subject repeated the test on 3 different days, whereupon the ingested substances were changed for each test at intervals of $1 \mathrm{wk}$ or so in accordance with the $3 \times 3$ Latin square method. We also instructed the subjects to prevent any changes in their own dietary habits with regard to dietary fiber, oligosaccharides, sugar alcohols or probiotics beginning from $1 \mathrm{wk}$ before the first test to the last test.

4. Analytical methods. Collected blood samples were centrifuged and subsequently left standing for 15 minutes before obtaining the serum samples. Serum glucose (22) and triacylglycerol (TG) were enzymatically determined with an automatic analyzer (DRI-CHEM 3500: Fujifilm, Tokyo, Japan). Analyses of serum free fatty acids (FFA) by an enzymatic method (23), insulin by an enzymatic immunoassay (24) and fructose by GCMS (25) were subcontracted to SRL Co. Ltd., (Tokyo, Japan). Serum acetate was analyzed by HPLC as fol-

Table 1. Composition of meals.

\begin{tabular}{lccr}
\hline & Meal at 21:00 (on the previous day) & Meal at 21:00, 16:00 (on the test day) & Total \\
\hline Energy $(\mathrm{kcal})$ & 515 & 695 & 1210 \\
Protein $(\mathrm{g})$ & 15.8 & 23.1 & 38.9 \\
Fat $(\mathrm{g})$ & 15.2 & 39.9 & 55.1 \\
carbohydrate $(\mathrm{g})$ & 79.1 & 61.3 & 140.4 \\
Dietary fiber $(\mathrm{g})$ & 4.8 & 3.2 & 8.0 \\
\hline
\end{tabular}

\footnotetext{
${ }^{1}$ Spaghetti with meat sauce+butter cookie.

${ }^{2}$ White bread + margarine + cheese.
}

Table 2. Physical characteristics of subjects.

\begin{tabular}{lccc}
\hline Physical parameter & DFAIII & Lactulose & Sucrose \\
\hline Height $(\mathrm{cm})$ & & $169.0 \pm 4.6$ & $59.2 \pm 0.5$ \\
Weight $(\mathrm{kg})$ & $59.7 \pm 3.6$ & $59.8 \pm 3.6$ & $20.7 \pm 0.5$ \\
Body mass index $\left(\mathrm{kg} / \mathrm{m}^{2}\right)$ & $20.8 \pm 0.6$ & $20.8 \pm 0.6$ & $108 \pm 5$ \\
Systolic blood pressure $(\mathrm{mmHg})$ & $108 \pm 4$ & $63 \pm 3$ & $64 \pm 4$ \\
Diastolic blood pressure $(\mathrm{mmHg})$ & $63 \pm 4$ & 6
\end{tabular}

Values are the means \pm SE $(n=8)$. There were no significant differences among subjects after ingestion of each sample with respect to physical characteristic parameters. 
lows: at first, serum samples were deproteinized with sulfosalicylic acid after adding crotonic acid as an internal standard and then extracted with diethyl ether, followed by addition of $0.2 \mathrm{M}$ sodium hydroxide solution as well as separation of the aqueous solution. HPLC was performed at $45^{\circ} \mathrm{C}$ on a pair of Shim-pack SCR-102H columns connected in series with a SCR-102H guard column (Shimadzu Co. Kyoto Japan). We used $5 \mathrm{nmol} / \mathrm{L}$ p-toluenesulfonic acid as the eluent. SCFA were detected by measurement of electrical conductivity (CDD-6A: Shimadzu Co.) using $5 \mathrm{mmol} / \mathrm{L}$ p-toluenesulfonic acid containing $100 \mu \mathrm{mol} / \mathrm{L}$ EDTA and $20 \mathrm{mmol} / \mathrm{L}$ Bis-Tris as the post-column buffer. Breath samples were collected within bags designed to obtain the alveolar air (GaSampler ${ }^{\circledR}$ : Quintron, Milwaukee, USA), and analyzed by gas chromatography (Microlyzer DP: Quintron) on the same day.

5. Statistical analysis. Data for serum glucose, fructose, and insulin levels from all subjects who completed this study were statistically analysed. The data on serum TG, FFA, acetate, breath hydrogen and methane of a few subjects who suffered diarrhea after ingestion of the samples were excluded from the statistical analysis. Values of the measured parameters are presented as the means $\pm S E(n=5-8)$. We determined significant differences at each elapsed time among the values after ingestion of the three test solutions, in parallel with the difference from the value of $0 \mathrm{~h}$ at each elapsed time for each test solution, by Sheffe's multiple comparison tests (Staview version 5.0, SAS Institute Inc. NC. USA). We regarded $P$ values less than 0.05 as a significant difference.

6. Ethical considerations. This study was approved by the institutional review board of the FANCL Corporation, and performed in accordance with the declaration of Helsinki.

\section{RESULTS}

Physical characteristics of the subjects taking part in this experiment throughout its period are presented in Table 2. There were no significant differences among the three groups in those respects.

After ingestion of DFAIII, a female subject suffered from watery diarrhea, while two female subjects had a loose passage and a male subject suffered from watery diarrhea after ingestion of lactulose. The same female subject suffered from diarrhea different in manifestation by ingestion of DFAIII and lactulose, respectively. No subjects suffered from diarrhea after ingestion of sucrose.

Serum glucose, fructose, and insulin levels were elevated at $0.5 \mathrm{~h}$ after sucrose ingestion as compared to those before ingestion. There were no changes in these parameters at the same times after DFAIII or lactulose ingestion (Fig. 2). Both serum glucose levels at $0.5 \mathrm{~h}$ after ingestion of DFAIII and lactulose were lower than that after sucrose ingestion. Similar tendencies were also observed for serum fructose levels. There was no significant difference at each time point between both cases of DFAIII and lactulose ingestion.
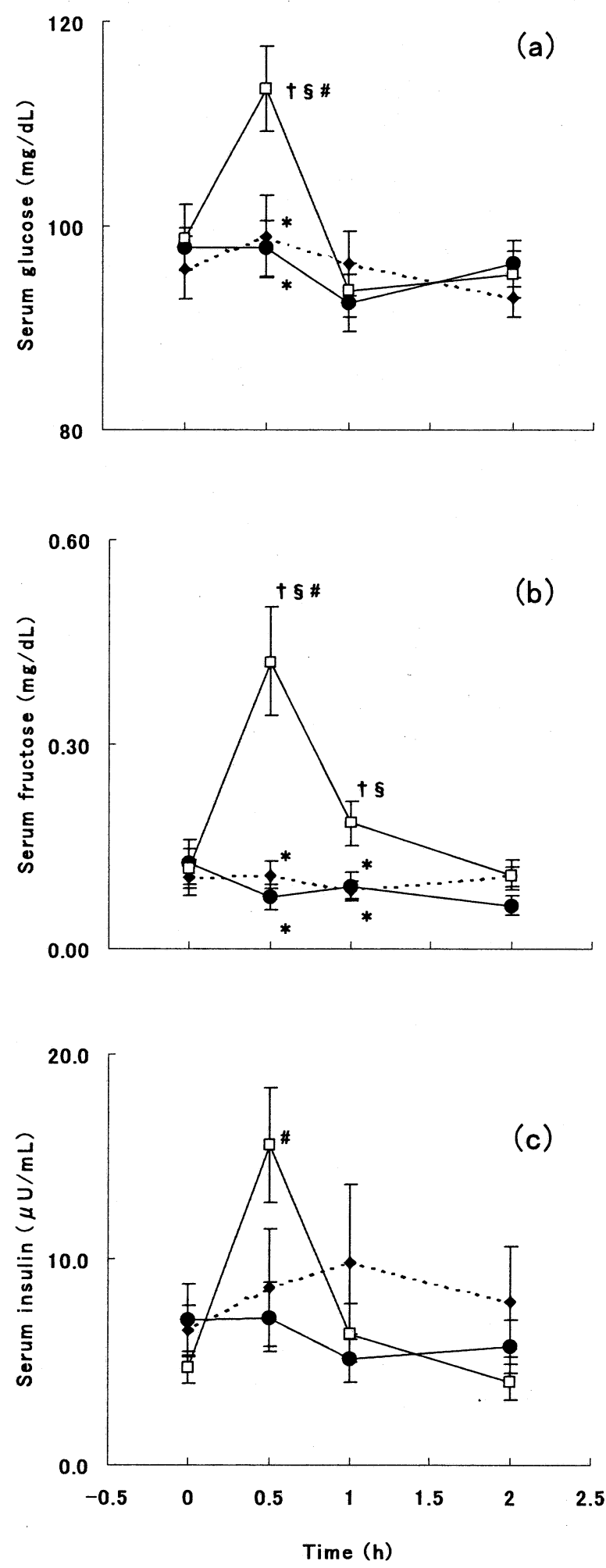

Fig. 2. Variation of serum glucose (a), fructose (b) and insulin (c) levels after ingestion of each sample. Each symbol and bar represents the means \pm SE ( $n=8$ except for $n=7$ at $1 \mathrm{~h}$ in the sucrose-ingested subject and at $2 \mathrm{~h}$ in the Iactulose-ingested subject); - — DFAIII, -.lactulose, - $\square-$ sucrose. Significantly different $(p<0.05)$; “*” from sucrose, " $\nmid$ " from lactulose, "§" from DFAIII at the same measured time, and "\#" from the value at time 0 by Sheffe's multiple comparison test. 


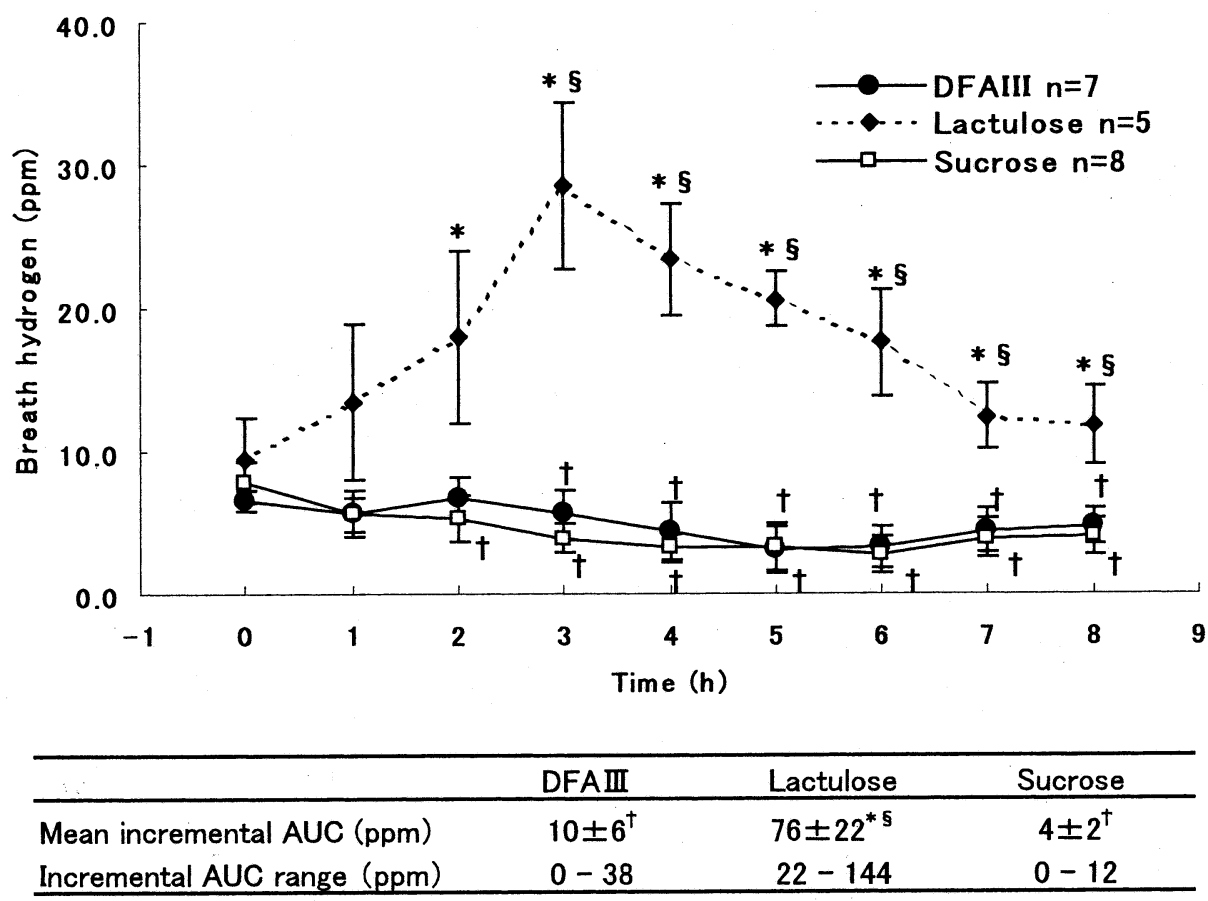

Fig. 3 Variation and incremental AUC of breath hydrogen after ingestion of each sample. Values are the means \pm SE $(n=5-$ $8)$; significantly different $(p<0.05)$; “*” from sucrose, " $\uparrow$ " from lactulose or " $§$ " from DFAIII at the same measured time by Sheffe's multiple comparison test.

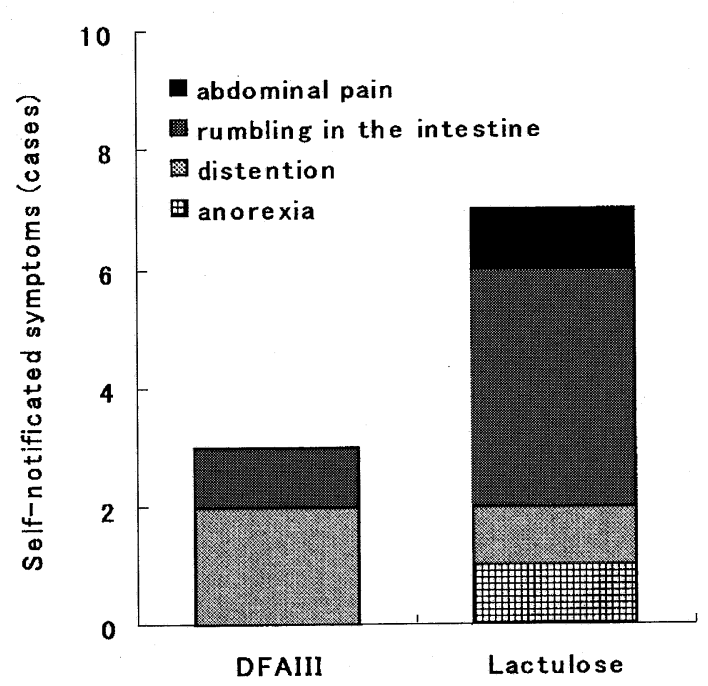

Fig. 4. Comparison of gastrointestinal symptom incidence in the DFAIII-ingested subjects with that in the lactulose-ingested subjects.

None of the subjects exhibited methane values over 2 ppm in their breath (data not shown). Breath hydrogen tended to increase gradually and reach a maximum at $3 \mathrm{~h}$ after ingestion of lactulose, while there were no changes up to $8 \mathrm{~h}$ in both cases of DFAIII and sucrose (Fig. 3). The difference in breath hydrogen between both cases of DFAIII and lactulose became significant at $3 \mathrm{~h}$, thenceforth lasting until $8 \mathrm{~h}$. The incremental area under the curve (AUC) for breath hydrogen over a period of $8 \mathrm{~h}$ was far low after ingestion of DFAIII or sucrose as compared to that after ingestion of lactulose. There were no changes in serum acetate, FFA, and TG levels in case of any disaccharide ingestion at all elapsed time points (data not shown). There was no significant difference in incremental AUC for acetate within $8 \mathrm{~h}$ after ingestion between DFAIII $(35.5 \pm 15.5 \mu \mathrm{mol} / \mathrm{L})$ and lactulose $(64.5 \pm 22.1 \mu \mathrm{mol} / \mathrm{L})$ or sucrose $(9.5 \pm 5.8 \mu \mathrm{mol} / \mathrm{L})$, but a significant difference at $p<0.05$ between lactulose and sucrose.

The subjects in case of DFAIII ingestion were less annoyed at gastrointestinal symptoms than in case of lactulose ingestion (Fig. 4). The incidence of abdominal pain and anorexia was actualized only when they ingested lactulose. Four subjects reported the incidence of rumbling in the intestine after ingestion of lactulose, whereas a subject or two complained of rumbling or distention after ingestion of DFAIII. These symptoms were regarded as mild or moderate. No subject seemed aware of any gastrointestinal symptoms after ingestion of sucrose. There were no other adverse events throughout this study.

\section{DISCUSSION}

The objective of this study was to examine the effects of DFAIII on sugar metabolism or enterobacterial fermentation in humans. We observed no changes in serum glucose, fructose, and insulin levels after ingestion of DFAIII in contrast to their elevations seen after ingestion of sucrose. It can be postulated from these results that DFAIII is an indigestible saccharide. Moreover, the subjects dosed with DFAIII showed no increase in breath hydrogen, which was in marked contrast to the increased hydrogen excretion in case of lactulose ingestion; indicating that DFAIII is not as fermentable as lactulose.

Indigestible saccharides pass through the small intestine and reach the colon in their intact form, which 
undergo fermentation by the acting of enterobacteria and produce hydrogen, methane, and SCFA. In this respect, DFAIII had a characteristic of the indigestible saccharides despite little fermentability relative to a familiar fermentable disaccharide "lactulose" $(16,17$, $19,26,27)$. Such an indigestible saccharide as reduced palatinose (12) seems to be almost completely fermented within $6 \mathrm{~h}$ in the human intestine (19). Also the increased breath hydrogen excretion occurs rapidly after ingestion of fructo-oligosaccharide (a mixture of tri- to penta-saccharides) (13), maltitol (reduced disaccharide) (14) and D-tagatose (monosaccharide) (15). The present study suggests that DFAIII may be the least fermentable of already known indigestible mono- or oligo-saccharides. The in vitro studies using the Ussing chamber technique have demonstrated that DFAIII directly enhances calcium absorption from the small and large intestines in its intact form $(8,9)$. Thus the relatively higher resistance of DFAIII to enterobacterial fermentation can put its stimulating effect on calcium absorption into practice. However, a previous animal experiment implies that the feeding of a 3\% DFAIII diet for $3 \mathrm{wk}$ induces higher SCFA levels and lower $\mathrm{pH}$ in the cecum than those seen with the diet deprived of DFAIII (7). Another study suggests that the ingestion of slightly digestible cornstarch causes no increases in breath hydrogen and serum acetate concentration within $8 \mathrm{~h}$ but elevates both parameters at $10-13 \mathrm{~h}$ than that seen with easily digestible starch (18). In this analogy, DFAIII fermentation may occur at later stages after its ingestion or exactly by adaptation of enterobacteria to DFAIII. The low fermentability of DFAIII in early stages after ingestion may possibly result from its unique structure with two glycoside linkages (Fig. 1). This possibility needs to be clarified in the future.

Although breath hydrogen level after ingestion of lactulose was significantly higher than that seen after ingestion of DFAIII or sucrose, there was no significant difference in serum acetate level between both cases at each elapsed time. There are a few published reports on an indication of peripheral venous acetate for monitoring events in the large bowel (20), although breath hydrogen seems to be more frequent. It has been demonstrated that blood acetate level increases after oral ingestion of $20 \mathrm{~g}$ of lactulose (16). In another study, the subjects who ingested $20 \mathrm{~g}$ of lactulose had a higher incremental AUC for serum acetate than those who ingested $20 \mathrm{~g}$ of sorbitol (17). Thus the dosage of lactulose adopted in this study may have been too low to increase peripheral serum acetate level especially in the 5 subjects not exhibiting increase after lactulose ingestion. However, the incremental change in AUC for serum acetate after ingestion of lactulose was higher than that seen after ingestion of sucrose; indicating that the parameter would reflect the fermentation of lactulose to a certain extent.

With the exception of a decreased tendency of FFA at $1 \mathrm{~h}$ after ingestion of sucrose, which is probably due to the raised blood sugar level, the FFA and TG levels remained unchangeable after ingestion of the samples; suggesting that the influences of the three sugars on lipid metabolism do not differ so much.

In our study, DFAIII exerted little adverse effects as compared with lactulose which has been lately used as prebiotic. Therefore, DFAIII is assumed to be relatively safe for use at a single dose.

The results of this study indicate that DFAIII is an indigestible and low fermentable sugar for humans in the early stages after its ingestion. DFAIII can serve as a valuable food material due to its low energy content.

\section{REFERENCES}

1) Li H-Y, Hagiwara H, Zhu W, Yokoyama C, Harada N. 1997. Isolation and NMR studies of di-D-fructose anhydride III from Lycoris radiata Herbert by supercritical extraction with carbon dioxide. Carbohyd Res 299: 301305.

2) Manley-Harris M, Richards GN. 1996. Di-D-fructose dianhydrides and related oligomers from thermal treatments of inulin and sucrose. Carbohyd Res 287: 183202.

3) Ratsimba V, García Fernández JM, Defaye J, Nigay H, Voilley A. 1999. Qualitative and quantitative evaluation of mono- and disaccharides in D-fructose, D-glucose and sucrose caramels by gas-liquid chromatography-mass spectrometry. Di-D-fructose dianhydrides as tracers of caramel authenticity. J Chromatogr A 844: 283-293.

4) Defaye J, García Fernández JM. 1995. The oligosaccharide components of caramels. Zuckerind 120: 700-704.

5) Yokota A, Hirayama S, Enomoto K, Miura Y, Tomita F. 1991. Production of inulin fructotransferase (depolymerizing) by Arthrobacter sp. H65-7 and preparation of DFAIII from inulin by the enzyme. J Ferment Bioeng 72: 258-261.

6) Suzuki T, Hara H, Kasai T, Tomita F. 1998. Effects of difructose anhydride III on calcium absorption in small and large intestine of rat. Biosci Biotechnol Biochem 62: 837-841.

7) Mitamura R, Hara H, Aoyama Y, Chiji H. 2002. Supplemental feeding of difructose anhydride III restores calcium absorption impaired by ovariectomy in rats. J Nutr 132: 3387-3393.

8) Mineo H, Hara H, Shigematsu N, Okuhara Y, Tomita F. 2002. Melibiose, difructose anhydride III and difructose anhydride IV enhance net calcium absorption in rat small and large intestinal epithelium by increasing the passage of tight junction in vitro. J Nutr 132: 3394 3399.

9) Mineo H, Hara H, Kikuchi H, Sakurai H, Tomita F. 2001. Various indigestible saccharides enhance net calcium transport from the epithelium of the small and large intestine of rats in vitro. J Nutr 131: 3243-3246.

10) Oku T. 1996. Available energy of nondigestible and/or nonabsorbable saccharides. Jpn J Nutr Diet 54: 143150 (Japanese).

11) Shouka・Kyusyu (Digestion•Absorption) 3rd Edition. (Hosoya N, Mutou Y, eds) 2003, p 266-270. Daiichi Shuppan, Tokyo, Japan (Japanese).

12) Fritz M, Siebert G, Kasper H. 1985. Dose dependence of breath hydrogen and methane in healthy volunteers after ingestion of a commercial disaccharide mixture, Palatinit. Br J Nutr 54: 389-400.

13) Stone-Dorshow T, Levitt MD. 1987. Gaseous response to ingestion of a poorly absorbed fructo-oligosaccharide 
sweetener. Am J Clin Nutr 46: 61-65.

14) Storey DM, Koutsou GA, Lee A, Zumbe A, Oliver P, Le Bot Y, Flourie B. 1998. Tolerance and breath hydrogen excretion following ingestion of maltitol incorporated at two levels into milk chocolate consumed by healthy young adults with and without fasting. J Nutr 128: 587-592.

15) Buemann B, Toubro S, Astrup A. 1998. D-Tagatose, a stereoisomer of D-fructose, increases hydrogen production in humans without affecting 24-hour energy expenditure or respiratory exchange ratio. J Nutr 128 : 1481-1486.

16) Pouteau E, Vahedi K, Messing B, Flourie B, Nguyen P, Darmaun D, Krempf M. 1998. Production rate of acetate during colonic fermentation of lactulose: a stableisotope study in humans. Am J Clin Nutr 68: $1276-$ 1283.

17) Rumessen JJ, Gudmand-Hoyer E. 1998. Fructans of chicory: intestinal transport and fermentation of different chain lengths and relation to fructose and sorbitol malabsorption. Am J Clin Nutr 68: 357-364.

18) Achour L, Flourie B, Briet F, Franchisseur C, Bornet F, Champ M, Rambaud JC, Messing B. 1997. Metabolic effects of digestible and partially indigestible cornstarch: a study in the absorptive and postabsorptive periods in healthy humans. Am J Clin Nutr 66: 1151-1159.

19) Cummings JH, Macfarlane GT, Englyst HN. 2001. Prebiotic digestion and fermentation. Am J Clin Nutr 73: 415S-420S.

20) Topping DL. Clifton PM. 2001. Short-chain fatty acids and human colonic function: roles of resistant starch and nonstarch polysaccharides. Physiol Rev 81: 10311064.

21) Alles MS, de Roos NM, Bakx JC, van de Lisdonk E, Zock PL, Hautvast GA. 1999. Consumption of fructooligosaccharides does not favorably affect blood glucose and serum lipid concentrations in patients with type 2 diabetes. Am J Clin Nutr 69: 64-69.

22) Takahashi Y, Kotaka Y, Takano R, Miyashita C, Amakawa T. 1984. Clinical evaluation of Fuji Dri-Chem (Glucose) System. Seibutsu Shiryo Bunseki 7: 56-61 (Japanese).

23) Sugo S, Matsumoto Y, Yamaoka T, Sakurabayashi I. 1990. Improved enzymatic method for determining free fatty acids in serum, with use of 3-octenoic acid. Clin Chem 36: 163.

24) Hara Y, Sakamoto T, Yamada E, Watanabe S, Kobayashi Y, Kobayashi S. 2000. Fundamental and clinical evaluation of insulin measurement using automatic chemiluminescence enzyme immunoassay analyzer, BCS600. Igaku to Yakugaku 44: 939-946 (Japanese).

25) Zhang S, Matsumoto I, Kuhara T. 1996. Simultaneous determination of sugars and sugar alcohols and its application in the study of diabetes mellitus by GC/MS.J Kanazawa Med Univ 21: 399-410.

26) Clausen MR, Jorgensen J, Mortenson PB. 1998. Comparison of diarrhea induced by ingestion of fructooligosaccharide Idolax and disaccharide lactulose: role of osmolarity versus fermentation of malabsorbed carbohydrate. Dig Dis Sci 43: 2696-2707.

27) Livesey G. 2001. Tolerance of low-digestible carbohydrates: a general view. Br J Nutr 85: S7-S16. 